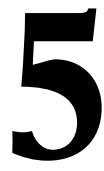

\title{
EMPLEO DE MOODLE EN LOS PROCESOS DE ENSEÑANZA-APRENDIZAJE DE DIRECCIÓN DE EMPRESAS: NUEVO PERFIL DEL ESTUDIANTE EN EL EEES
}

\author{
(USING MOODLE IN TEACHING-LEARNING PROCESSES IN BUSINESS \\ MANAGMENT: THE NEW PROFILE OF EHEA STUDENT)
}

Antonio Padilla-Meléndez, Ana Rosa del Águila-Obra y Aurora Garrido-Moreno Universidad de Málaga

DOI: 10.5944/educXX1.18.1.12314

Cómo referenciar este artículo/How to reference this article:

Padilla Meléndez, A.; Del Águila Obra, A. R. y Garrido Moreno, A. (2015). Empleo de Moodle en los procesos de enseñanza-aprendizaje de Dirección de Empresas: nuevo perfil del estudiante en el EEES. Educación XX1, 18(1), 125-146. doi: 10.5944/educXX1.18.1.12314

Padilla Meléndez, A.; Del Águila Obra, A. R. y Garrido Moreno, A. (2015). Using Moodle in teaching-learning processes in business management: the new profile of EHEA student. Educación XX1, 18(1), 125-146. doi: 10.5944/educXX1.18.1.12314

\section{RESUMEN}

Este trabajo analiza el uso de la plataforma Moodle para mejorar los procesos de enseñanza-aprendizaje en diversas asignaturas de Dirección de Empresas en el contexto del Espacio Europeo de Educación Superior (EEES). Con los nuevos grados se demanda que el/la alumno/a cada vez tenga más competencias transversales relacionadas con sus habilidades de comunicación y de uso de las tecnologías de la información (TI). El objetivo que persigue este trabajo es analizar cómo los estudiantes perciben el uso de las TI como herramientas de aprendizaje, cuáles son las variables que determinan su aceptación y uso, así como observar si existen diferencias significativas en estos aspectos entre los alumnos de titulaciones de Diplomatura/Licenciatura (plan antiguo) y de Grado. Para ello, se realizó un estudio empírico durante tres cursos académicos con una muestra de 386 estudiantes, de los cuales se recopiló información a través de un cuestionario basado en web. Con los datos obtenidos se realizaron análisis estadísticos univariantes y bivariantes y se estimaron diversos modelos de ecuaciones estructurales. Los principales resultados muestran la importancia de que los estudiantes perciban la utilidad del uso de la tecnología, así como su facilidad de uso y que se trate de un entorno que sea, al mismo tiempo, entretenido. Respecto a las principales 
diferencias observadas, los estudiantes de Grado mostraron una mayor intención de uso de la plataforma y una mayor experiencia en el uso de las TI. Como principales conclusiones destaca cómo en el contexto del EEES, la enseñanza universitaria presencial apoyada por la tecnología (blended learning) supone un nuevo escenario de aprendizaje y ante este nuevo contexto el docente debe tomar un papel más activo en el proceso, desarrollando roles como el de gestor de contenidos y el de gestor de una comunidad.

\section{PALABRAS CLAVE}

Innovación educativa, tecnología educativa, experiencia educativa, campus virtual, rol del docente.

\section{ABSTRACT}

This paper discusses the use of the Moodle platform to improve the teaching-learning processes in several subject areas of Business Management in the context of the European Higher Education Area (EHEA). With the new degrees, there will be a demand for students with increasingly more transferable skills related to communication skills and use of information technology (IT). The objective of this paper is to analyze how students perceive the use of IT learning tools, what the main variables are that determine its acceptance and use, and to examine if there are significant differences in these aspects between students of the diploma/bachelor's degree and the new degrees (EHEA). With this purpose, an empirical study was carried out for three academic years with a sample of 386 students. The information was collected through a web-based questionnaire. Univariate and bivariate statistical analyses were carried out and several structural equation models were estimated. The results demonstrate that it is important that students perceive the usefulness and ease of use of technology and, at the same time, the entertaining side of the system. The differences noted were that students from the new degrees show a greater intention to use the platform and greater experience using IT. As main conclusions, it is highlighted that in the context of the EHEA, the blended learning system means a new stage of learning, and in this new context, teachers must take a more active role in the learning process, developing new roles such as content and community managers.

\section{KEY WORDS}

Educational innovation, educational technology, educational experience, virtual classrooms, teacher role. 


\section{INTRODUCCIÓN}

La utilización de la tecnología en el ámbito de los procesos de enseñanza-aprendizaje universitarios se ha extendido en los últimos años y se valora como un factor importante para la mejora de las prácticas educativas en sus diferentes niveles. Esta utilidad está probada para las enseñanzas superiores y las áreas aplicadas, tales como la Dirección de Empresas (Martins y Kellermanns, 2004).

Con la implementación de los nuevos Grados, basados en el Espacio Europeo de Educación Superior (EEES) cabe pensar que el uso de esta tecnología no hará más que crecer, ya que se demandan nuevas competencias al alumnado relacionadas con sus habilidades de comunicación y su competencia tecnológica, con lo que el uso de la tecnología como apoyo a la docencia, en este marco del EEES, permite además avanzar en el desarrollo de las competencias del estudiante. Existen publicaciones que analizan la introducción de nuevas metodologías en la enseñanza de la Dirección de Empresas, entre otros, se ha estudiado el uso de simuladores en el ámbito universitario (Arias, Haro, Romerosa y Navarro-Paule, 2010) o la aceptación de la tecnología por estudiantes de esta misma disciplina (Martins y Kellermanns, 2004; Padilla-Meléndez, Garrido-Moreno y del Águila-Obra, 2008). Sin embargo, aún siguen existiendo necesidades de investigación relacionadas con el EEES (Florido, Jiménez y Santana, 2011) y el grado de aceptación de la tecnología por parte de los estudiantes. De hecho Gómez-García, Ramiro, Ariza y Reina-Granados (2012), en un completo análisis bibliométrico realizado en la presente revista, observaron cómo el análisis de la implantación de nuevas tecnologías en la educación puede considerarse un área de investigación relevante, aunque el número de trabajos publicados sobre la cuestión es aún escaso, por lo que es necesario seguir profundizando en su estudio. Además, específicamente en el ámbito de la educación superior de futuros directivos, este tema es clave, ya que el estudiante trabajará en un entorno organizativo (empresas u otras instituciones) donde la tecnología, particularmente las TI, juegan un papel determinante.

En este trabajo se analizan diversos aspectos del empleo de dichas tecnologías, y se aporta evidencia empírica sobre el efecto que la incorporación de los nuevos grados está teniendo en la aceptación y uso de la tecnología, en particular de alumnos de Dirección de Empresas. Para ello, se utiliza el modelo de aceptación de la tecnología, ampliamente aceptado y citado en la literatura (Hsiao y Yang, 2011), que incluye variables tales como la utilidad percibida de una tecnología concreta, la facilidad de uso percibida, la actitud hacia la misma y la intención de uso. Para contrastar dicho modelo, se analizan datos de varios proyectos de innovación educativa que, con el objetivo general de adaptar diversas asignaturas al EEES, ha desarrollado un 
equipo de profesores del área de Organización de Empresas de una universidad media española, en los cursos académicos 2008/09, 2009/10 y 2010/11. En dichos proyectos se han visto implicados un total de 1.290 alumnos, de los cuales se ha recopilado información a través de un cuestionario basado en web, obteniendo una muestra de 386 estudiantes. El análisis de estos datos permite extraer conclusiones relevantes sobre la utilidad y efectos de los proyectos de innovación educativa realizados.

El artículo continúa con una explicación breve de los antecedentes y fundamentación teórica de la experiencia, una descripción de la metodología seguida, el análisis de resultados, su discusión y las principales conclusiones.

\section{ANTECEDENTES Y FUNDAMENTACIÓN TEÓRICA}

\section{Nuevas tecnologías en los procesos de enseñanza/aprendizaje}

El desarrollo de internet y las redes sociales en los últimos años ha hecho que profesores y alumnos empleen cada vez más las TI en los procesos de enseñanza/aprendizaje. Con objeto de formar a los ciudadanos para vivir en la sociedad del conocimiento, uno de los objetivos de los distintos países en la última década ha sido la integración de las TI en el sistema educativo (Suárez-Rodríguez, Almerich, Gargallo-López y Aliaga, 2013). En ocasiones, se ha dicho que los estudiantes están por delante de los profesores en el empleo de estas herramientas, toda vez que, en general, son una generación que ha convivido con la tecnología, por ejemplo usando videojuegos. De hecho, es la denominada generación Net, o Millennials, que utilizan las TI sin ningún tipo de reserva o miedo, como nunca nadie lo había hecho, se trata de la primera generación que ha crecido con las facilidades de comunicación de internet y están tan acostumbrados a su uso como sus padres lo estaban al de los teléfonos (Greenberg, 2010). Por tanto, es una generación proactiva, que se comunica con tecnología de forma natural y que espera que se le proporcionen las herramientas adecuadas para ello, también en el entorno educativo.

Esta división digital habría provocado que los estudiantes sean miembros de redes sociales o empleen herramientas de mensajería instantánea en una proporción mayor que los profesores universitarios. Además, las TI son un factor importante para la mejora de las prácticas educativas, en particular, está probado que la tecnología es valiosa en enseñanzas superiores y en áreas aplicadas, tales como la Dirección de Empresas (Ives y Jarvenpaa, 1996). En este sentido, se han desarrollado dos enfoques diferentes para el uso de las TI en educación, el e-learning y el blended learning (b-learning). 
Como es conocido, el primer enfoque implica una enseñanza/aprendizaje no presencial, empleando TI. El segundo enfoque consiste en el empleo de las TI como complemento a la enseñanza presencial. En este caso, las TI (principalmente internet, a través de las plataformas basadas en la web, o también plataformas e-learning) han sido utilizadas principalmente para promover una mayor interacción entre profesores y estudiantes, y mejorar el contacto entre estos, siendo un complemento a la enseñanza/aprendizaje presencial, y consiguiendo, además, ahorro de tiempo (Lonn y Teasley, 2009).

Esta incorporación de las nuevas tecnologías a las aulas está generando nuevos escenarios de aprendizaje y transformando de manera radical el quehacer docente (García-Varcárcel y Tejedor-Tejedor, 2011), lo que requiere el desarrollo de nuevos roles y habilidades por parte del docente, tales como el gestor de contenidos y gestor de una comunidad (community manager) (Lara, 2005; Martínez, Fossas y Cobo, 2010). El gestor de contenidos es la persona encargada de diseñar y gestionar (publicar, mantener y actualizar) contenidos multimedia publicados en un sitio web. El community manager, por su parte, es un rol que consiste en el manejo de internet y las nuevas tecnologías e implica ser un experto/a en social media, escribiendo de forma creativa, siendo capaz de construir, hacer crecer, gestionar y dinamizar comunidades alrededor de algún tema/actividad, comunicándose eficazmente por medios electrónicos con los usuarios.

Respecto a las plataformas de enseñanza/aprendizaje basadas en internet, existen una gran diversidad de ellas, tanto comerciales como de código abierto. En las experiencias de innovación educativa que se analizan en este estudio se ha empleado Moodle, que contaba en abril de 2014 con 8.054.294 de cursos y 74.618.581 de usuarios, de los cuales 1.163.014 eran profesores, de 238 países, siendo España el segundo país en el ranking después de Estados Unidos (Moodle, 2014). Otro indicador de que el uso de la plataforma Moodle se encuentra consolidado en España se visualiza en los estudios académicos que han abordado esta cuestión recientemente, tales como Martín-Blas y Serrano-Fernández (2009) y Romero, Espejo, Zafra, Romero y Ventura (2013).

\section{El modelo de aceptación de la tecnología (TAM)}

El modelo de aceptación de la tecnología es ampliamente conocido (Venkatesh, 2000; Venkatesh, Speier y Morris, 2002) y se han realizado diversos estudios aplicándolo al ámbito educativo (por ejemplo, Martins y Kellermanns, 2004). El modelo básico contempla cómo la utilidad percibida de una herramienta tecnológica (grado en que una persona cree que el uso de TI aumentará el rendimiento de su trabajo) y su facilidad de uso (grado 
en que una persona cree que su uso está libre de esfuerzo), afectan a la actitud de uso de dicha tecnología.

El modelo TAM fue inicialmente desarrollado por Davis (1989), a partir de los fundamentos de la teoría de la acción razonada (Fishbein y Ajzen, 1975). El TAM supone que la adopción y el uso vienen determinados por la intención de utilizar un sistema, que a su vez está afectada por la utilidad percibida, la facilidad y las actitudes hacia el uso. En consecuencia, la utilidad percibida y la facilidad de uso percibida son los dos principales predictores de la aceptación y el uso. El modelo inicial fue mejorado posteriormente, mediante la adición de otras variables relevantes, así, el denominado TAM2 incorpora otras construcciones teóricas, incluyendo los procesos de influencia social y los procesos cognitivos instrumentales (Venkatesh y Davis, 2000). Y el TAM3 plantea nuevas relaciones teóricas, tales como los efectos moderadores de experiencia en las relaciones clave. Esta versión extendida sugiere que la experiencia será el moderador de las relaciones entre la facilidad de uso y la utilidad percibida, la ansiedad y la facilidad de uso percibida, la percepción de facilidad de uso y la intención conductual (Venkatesh y Bala, 2008).

El modelo TAM ha recibido un fuerte apoyo teórico y empírico en la literatura y ha sido aplicado de manera consistente durante los últimos 25 años (Schoonenboom, 2014). De hecho, Hsiao y Yang (2011), en base a un extenso análisis bibliométrico, resaltan la validez y consistencia del modelo para analizar la aceptación y uso de sistemas de información, y destacan que el mismo ha recibido más de 2.000 citas en revistas académicas. En consecuencia, este marco teórico se considera ahora apropiado por algunos autores para predecir la satisfacción del estudiante en contextos de aprendizaje, ya que se ha demostrado que las variables TAM influyen significativamente en la satisfacción del estudiante en estos entornos (Arbaugh et al., 2009).

Por otra parte, una variable adicional que está siendo recientemente añadida al modelo TAM es el entretenimiento. Los usuarios no son siempre racionales o lógicos y la emoción juega un papel importante en la aceptación, por parte de los usuarios, de una determinada tecnología (Zhang y Li, 2005), luego el entretenimiento debe ser considerado en un modelo de aceptación de la tecnología. El entretenimiento o disfrute percibido es el grado en que "la actividad de uso de un sistema específico se percibe como agradable en sí, al margen de las consecuencias de rendimiento resultantes del uso del sistema» (Venkatesh, 2000, p. 351), siendo una variable compleja que incluye el placer individual, la estimulación psicológica y los intereses. Además, el entretenimiento se considera una creencia (Lee, Cheung y Chen, 2005) y representa una motivación intrínseca asociada con el uso de cualquier sistema nuevo (Venkatesh y Bala, 2008). Resumiendo la investigación sobre la percepción del entretenimiento, esta variable ha sido analizada desde diver- 
sos puntos de vista (Venkatesh, 2000), y se han estudiado sus antecedentes (Chung y Tan, 2004), su relación con la satisfacción del usuario (Lin, Ray y Tsai, 2005) y en el contexto del modelo TAM, se ha considerado un motivador intrínseco (Ahn, Ryu y Han, 2007; Cheong y Park, 2005; Liao y Tsou, 2009; Oh et al., 2009; Venkatesh, 2000; Venkatesh y Bala, 2008), así como el resultado de la facilidad de uso (Moon y Kim, 2001). En este sentido, varios estudios han probado la relevancia empírica del aspecto lúdico como una variable de integración del modelo TAM. Por todo ello, en el presente estudio se incluyó la variable entretenimiento como variable explicativa del modelo.

\section{METODOLOGÍA}

\section{Metodología de uso de Moodle}

La experiencia que se describe ha tenido el objetivo de mejorar el proceso de enseñanza-aprendizaje de la materia de Dirección de Empresas y ha consistido en emplear durante tres cursos académicos diversas funcionalidades de la plataforma de fuentes abiertas Moodle, en diversas asignaturas de Dirección de Empresas, que se ofertaban en diferentes centros y cursos de una universidad española. La originalidad de esta experiencia se encuentra en la variedad de funcionalidades utilizadas, y en el desempeño de los nuevos roles por parte del docente, partiendo de la consideración de que la plataforma por sí sola no es nada, sin la labor activa del mismo. En la plataforma Moodle las asignaturas se organizaron por bloques separados:

1. Bloque inicial con información sobre la asignatura e información sobre el/la profesor/a: programa de la asignatura, instrucciones para trabajos en grupo, listados de notas, programación temporal de contenidos, sistema de evaluación y breve currículo de los docentes. Con esta opción se reduce la asimetría de la información, entre los alumnos, dado que todos los estudiantes disponían de la misma información actualizada en todo momento, aunque se hubiera producido su ausencia en el aula, por enfermedad u otros motivos.

2. Bloque de comunicación/participación: herramientas de comunicación y colaboración, sistema de tutorías programadas, tutoría virtual, calendario de eventos, foros como sistema para la inscripción de trabajos y wikis para los trabajos en grupo. El foro de tutoría virtual fue una de las herramientas mejor valorada por los alumnos, ya que permitía la resolución de dudas (tanto por el docente como por otros alumnos), mantener informados a todos sobre la evolución de la asignatura (cambios en horarios, disponibilidad de 
materiales para su descarga, etc.). La gestión de este bloque está relacionada con el rol del profesor/a como community manager.

3. Bloque de contenido: materiales del temario del programa de cada asignatura y contenidos prácticos asociados a dichos temas. La gestión de este bloque se refiere especialmente al rol de gestor de contenidos desempeñado por los docentes. Los sub-bloques fueron los siguientes:

a) Contenidos docentes: los estudiantes disponían del material tanto teórico como práctico antes de su análisis en el aula, lo que les permitía seguir de un modo más distendido las lecciones magistrales. En las clases prácticas, los alumnos contaban con los casos de estudio y ejercicios a realizar en grupo en el aula.

b) Recursos externos (videos, lecturas, informes, referencias online): fueron recopilados por los profesores y alumnos implicados en el proyecto a modo de materiales complementarios. También se enlazaron juegos, tales como Café World (de administración de un restaurante) y Roller Coaster Tycoon (de gestión empresarial de un parque de atracciones).

Además, se incluyeron bloques de envío de trabajos (con la herramienta tareas), de consulta (para que los alumnos valorasen actividades a realizar en la asignatura) y encuestas (para recopilar datos sobre la percepción de las funcionalidades de la plataforma por parte de los estudiantes).

\section{Metodología de evaluación de la experiencia}

Con el objetivo de evaluar la experiencia se realizó una encuesta web administrada a través de la herramienta de encuestas de Moodle para cada asignatura. En dicha encuesta se preguntaba al alumno/a sobre la frecuencia de uso de ciertas TI con fines formativos, así como sobre sus percepciones en relación al uso de la tecnología, en particular en relación a internet $\mathrm{y}$ al aula virtual. El cuestionario fue confeccionado utilizando escalas basadas en la literatura, y previamente validadas (Byoung-Chan, Jeong-Ok e In, 2009; Padilla-Meléndez et al., 2008; Terzis y Economides, 2011). Para la medición de las distintas variables se utilizaron escalas Likert con una puntuación de 1 a 7, desde la opción completamente en desacuerdo hasta completamente de acuerdo, siendo la posición intermedia indiferente.

El cuestionario fue enviado a una población de 1.290 alumnos que estaban matriculados en las distintas asignaturas que formaron parte de la 
experiencia. Se obtuvo una muestra efectiva de 386 , lo que supone una tasa de respuesta del 29,92 por ciento.

Una vez observado que los datos recogidos eran adecuados para la aplicación del análisis factorial, y como una forma de evaluar la escala de medida, fueron analizados cuatro aspectos básicos de la misma (Hair, Anderson, Tatham y Black, 2004): su definición conceptual, su validez y fiabilidad y su dimensionalidad. En cuanto a la definición conceptual, la escala fue construida basándose en un amplio análisis de la literatura. Por otra parte, la validez de una medida hace referencia al grado en que tal proceso de medida está libre de error. En relación a la escala propuesta, fue comprobada su validez considerando las distintas modalidades del concepto (de contenido, constructo, convergente, discriminante y externa). Para analizar la fiabilidad de la escala, se utilizó el alfa de Cronbach siendo cercano a 0,9 en todos los casos, con lo que se comprobó la fiabilidad de la escala. Por último, para analizar su dimensionalidad se efectuó un análisis factorial exploratorio utilizando el método de componentes principales, y se obtuvo un modelo factorial que efectivamente validaba la estructura de factores propuestas a nivel teórico.

Tras comprobar la validez y fiabilidad de la escala, se realizaron análisis estadísticos univiantes y bivariantes, y se siguió una metodología de ecuaciones estructurales, para contrastar el modelo TAM con los datos obtenidos. Dicha metodología permite evaluar la idoneidad del modelo teórico bajo estudio respecto a los datos empíricos, y examinar la significación de relaciones específicas. Dado que los datos no eran normales (el estimador de Mardia normalizado de la curtosis multivariante era igual a 17,37 superior al valor recomendado de 1,96), para la estimación del modelo de ecuaciones estructurales se utilizó el programa estadístico EQS 6.1. Este programa permite estimar indicadores robustos de bondad del ajuste, así como el estadístico chi-cuadrado robusto (Satorra-Bentler scaled statistics), que corrige la chi-cuadrado teniendo en cuenta la no normalidad de las variables (Satorra y Bentler, 2001).

\section{RESULTADOS}

Como se indicó anteriormente, se recabaron datos durante tres cursos académicos, siendo un 57,3 por ciento de los encuestados alumnos de Diplomatura/Licenciatura, mientras que un 42,7 por ciento eran alumnos de nuevos títulos de Grado. Un 45,1 por ciento de los estudiantes encuestados eran hombres y un 54,9 por ciento mujeres y cursaban estudios en siete centros (facultades y/o escuelas) distintos de la Universidad (Tabla 1). 


\begin{tabular}{|l|c|c|}
\hline \multicolumn{1}{|c|}{ Ítem } & Frecuencia & Porcentaje \\
\hline Curso académico & & \\
- 2008/2009 (Diplomatura/Licenciatura) & 128 & 33,2 \\
- 2009/2010 (Diplomatura/Licenciatura) & 93 & 24,1 \\
- 2010/2011(Grados) & 165 & 42,7 \\
Género & 174 & \\
- Masculino & 212 & 54,1 \\
- Femenino & & \\
Facultad o Escuela & 111 & 28,8 \\
- Estudios Sociales y del Trabajo & 102 & 26,4 \\
- Turismo & 64 & 16,6 \\
- Técnica Superior de Ingeniería Informática & 47 & 12,2 \\
- Económicas & 33 & 8,5 \\
- Técnica Superior de Ingeniería en Telecomuni- & & \\
$\quad$ caciones & 21 & 5,4 \\
- Politécnica & 8 & 2,1 \\
\hline
\end{tabular}

Tabla 1. Variables demográficas y descripción de la muestra

Respecto a las variables analizadas sobre aceptación y uso de la plataforma Moodle, se observó que las variables que registraron mayores puntuaciones medias, en estudiantes de los distintos planes de estudio, estaban relacionadas con la facilidad de uso del sistema («El campus virtual es fácil de usar»). Por otra parte, la variable que registró una valoración más baja por parte de los estudiantes fue el entretenimiento ( Me divierto usando el campus virtual», «Encuentro el campus virtual entretenido») (Tabla 2).

\begin{tabular}{|l|l|c|c|c|c|}
\hline \multicolumn{2}{|c|}{ Ítem } & \multicolumn{2}{c|}{$\begin{array}{c}\text { Diplomatura/ } \\
\text { licenciatura }\end{array}$} & \multicolumn{2}{c|}{ Grado } \\
\cline { 2 - 6 } \multicolumn{2}{|c|}{} & Media & $\begin{array}{c}\text { Desviación } \\
\text { típica }\end{array}$ & Media & $\begin{array}{c}\text { Desviación } \\
\text { típica }\end{array}$ \\
\hline \multirow{2}{*}{ Entretenimiento } & $\begin{array}{l}\text { E1 - Me divierto usando } \\
\text { el campus virtual }\end{array}$ & 4,08 & 1,161 & 3,53 & 1,516 \\
\cline { 2 - 6 } & $\begin{array}{l}\text { E2 - Encuentro el cam- } \\
\text { pus virtual entretenido }\end{array}$ & 4,47 & 1,309 & 3,81 & 1,588 \\
\cline { 2 - 6 } & $\begin{array}{l}\text { E3 - Me gusta usar el } \\
\text { campus virtual }\end{array}$ & 5,41 & 1,268 & 4,61 & 1,468 \\
\hline
\end{tabular}




\begin{tabular}{|c|c|c|c|c|c|}
\hline & \multirow{2}{*}{ Ítem } & \multicolumn{2}{|c|}{$\begin{array}{c}\text { Diplomatura/ } \\
\text { licenciatura }\end{array}$} & \multicolumn{2}{|c|}{ Grado } \\
\hline & & Media & $\begin{array}{c}\text { Desviación } \\
\text { típica }\end{array}$ & Media & $\begin{array}{l}\text { Desviación } \\
\text { típica }\end{array}$ \\
\hline \multirow{4}{*}{$\begin{array}{l}\text { Utilidad } \\
\text { percibida }\end{array}$} & $\begin{array}{l}\text { UP1 - El campus virtual } \\
\text { mejora mis resultados } \\
\text { de aprendizaje (rendi- } \\
\text { miento) }\end{array}$ & 5,43 & 1,356 & 4,94 & 1,333 \\
\hline & $\begin{array}{l}\text { UP2 - El campus virtual } \\
\text { es muy útil para mí }\end{array}$ & 5,78 & 1,362 & 5,65 & 1,219 \\
\hline & $\begin{array}{l}\text { UP3 - El campus virtual } \\
\text { me ayuda a desarrollar } \\
\text { mi aprendizaje de mane- } \\
\text { ra efectiva }\end{array}$ & 5,23 & 1,397 & 5,00 & 1,302 \\
\hline & $\begin{array}{l}\text { UP4 - El uso del campus } \\
\text { virtual facilita mi trabajo } \\
\text { en esta asignatura }\end{array}$ & 5,72 & 1,339 & 5,68 & 1,205 \\
\hline \multirow{4}{*}{$\begin{array}{l}\text { Facilidad de uso } \\
\text { percibida }\end{array}$} & $\begin{array}{l}\text { FUP1 - Es fácil emplear } \\
\text { el aula virtual para aque- } \\
\text { llo que necesito hacer }\end{array}$ & 5,69 & 1,249 & 5,72 & 1,119 \\
\hline & $\begin{array}{l}\text { FUP2 - El campus vir- } \\
\text { tual es fácil de usar } \\
\end{array}$ & 6,06 & 1,003 & 6,07 & 1,200 \\
\hline & $\begin{array}{l}\text { FUP3 - Mi interacción } \\
\text { con el campus virtual es } \\
\text { clara y comprensible }\end{array}$ & 5,86 & 1,151 & 5,72 & 1,192 \\
\hline & $\begin{array}{l}\text { FUP4 - Es sencillo llegar } \\
\text { a ser un experto en el } \\
\text { uso del campus virtual }\end{array}$ & 5,57 & 1,297 & 5,69 & 1,333 \\
\hline Intención de uso & $\begin{array}{l}\text { IU1 - Tengo intención de } \\
\text { usar el campus virtual } \\
\text { con bastante frecuencia } \\
\text { en el curso siguiente }\end{array}$ & 5,27 & 1,357 & 6,05 & 1,248 \\
\hline
\end{tabular}

Tabla 2. Estadísticos descriptivos

Asimismo, se aplicó la diferencia de medias para analizar de forma exploratoria las diferencias entre los estudiantes de titulaciones de Diplomatura/Licenciatura y las de Grado (Tabla 3). Se obtuvieron diferencias significativas en diversos ítems de las variables entretenimiento, utilidad percibida e intención de uso.

\begin{tabular}{|l|l|l|c|c|}
\hline \multicolumn{1}{|c|}{ Ítem } & $\begin{array}{c}\text { U de Mann- } \\
\text { Whitney }\end{array}$ & $\begin{array}{c}\text { W de } \\
\text { Wilcoxon }\end{array}$ & $\begin{array}{c}\text { Significación } \\
\text { (bilateral) }\end{array}$ & $\begin{array}{c}\text { Diferencia } \\
\text { de medias }\end{array}$ \\
\hline E1 & 14843,5 & 28538,5 & 0,001 & Significativa \\
\hline E2 & 14065,5 & 27760,5 & 0,000 & Significativa \\
\hline E3 & 12572 & 26267 & 0,000 & Significativa \\
\hline
\end{tabular}




\begin{tabular}{|l|l|l|c|c|}
\hline \multicolumn{1}{|c|}{ Ítem } & $\begin{array}{c}\text { U de Mann- } \\
\text { Whitney }\end{array}$ & $\begin{array}{c}\text { W de } \\
\text { Wilcoxon }\end{array}$ & $\begin{array}{c}\text { Significación } \\
\text { (bilateral) }\end{array}$ & $\begin{array}{c}\text { Diferencia } \\
\text { de medias }\end{array}$ \\
\hline UP1 & 14170 & 27865 & 0,000 & Significativa \\
\hline UP2 & 16579 & 30274 & 0,112 & \\
\hline UP3 & 16252,5 & 29947,5 & 0,059 & \\
\hline UP4 & 17369,000 & 31064,000 & 0,406 & \\
\hline FUP1 & 18116,500 & 31811,500 & 0,911 & \\
\hline FUP2 & 17213,000 & 41744,000 & 0,313 & \\
\hline FUP3 & 16883,000 & 30578,000 & 0,190 & \\
\hline FUP4 & 16851,000 & 41382,000 & 0,185 & \\
\hline IU1 & 11094,500 & 35625,500 & 0,000 & Significativa \\
\hline
\end{tabular}

Tabla 3. Diferencias de medias

Con objeto de contrastar si las relaciones planteadas por el modelo de aceptación de la tecnología se contrastaban en la muestra utilizada, y comparar asimismo si se habían producido diferencias en cuanto a la aceptación y uso de tecnologías con la llegada de los nuevos Grados, se estimaron dos versiones del modelo TAM, incluyendo la variable entretenimiento, utilizando ecuaciones estructurales. Se planteó un primer modelo «estudiante pre-EEES», con los datos de 2008/2009 y un segundo modelo «estudiante EEES», con los datos del periodo 2010/2011. Se comprobó la bondad del ajuste de ambos modelos utilizando diversos indicadores. Respecto a la bondad de ajuste del modelo global, se observó que aunque la chi-cuadrado era no significativa $(\mathrm{p}<0,05)$, el resto de índices considerados mostraban un ajuste satisfactorio, superando los límites recomendados (véase Tablas 4 y 5). El ajuste del modelo de medida también fue satisfactorio, y respecto al modelo estructural, se observó que todos los coeficientes estimados en dichas ecuaciones eran significativos. Con todo ello, se dio el ajuste del modelo como satisfactorio.

En los gráficos 1 y 2 aparece representada la estimación de ambos modelos, sus coeficientes de regresión estandarizados y la significatividad de las relaciones planteadas. Respecto a las relaciones observadas, en ninguno de los dos modelos el impacto del entretenimiento en la intención de uso fue significativo. Solo mostró un impacto indirecto a través de la actitud. Este hecho puede deberse a que al estar analizando el caso de una tecnología de uso obligado (para superar la asignatura), el entretenimiento no determina directamente el uso de la misma, sino que fomenta una actitud positiva hacia la plataforma, que sí incide en su intención de uso.

Asimismo, en el modelo estimado con datos de alumnos de Grado (estudiantes EEES) se observa que la facilidad de uso no muestra un impacto en la actitud, lo que puede deberse a que son alumnos más experimentados 
en el uso de TI. Se observa en este periodo que la facilidad de uso solo impacta en la actitud a través de la utilidad percibida. Además, el impacto del entretenimiento en la actitud es mucho mayor en este último periodo $(0,425$ frente a 0,185 ) lo que puede indicar que dicha variable juega un papel cada vez más relevante en la actitud de los alumnos.

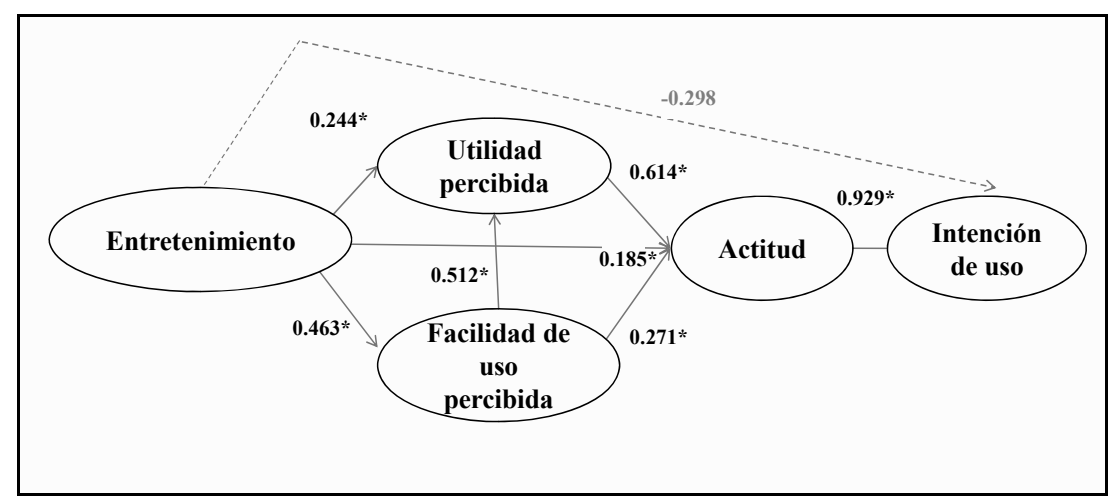

Gráfico 1. Modelo estimado «estudiante pre-EEES» ( $\mathrm{N}=128$ casos)

\begin{tabular}{|l|c|c|}
\hline \multicolumn{1}{|c|}{ Índice } & Valor & Valor recomendado \\
\hline Chi-cuadrado de Satorra-Bentler & $\mathrm{P}=0,15$ & $\mathrm{p} \geq 0,05$ \\
\hline RMSEA & 0,039 & $\leq 0,05$ \\
\hline Intervalo de confianza del RMSEA & $(0,00,0,071)$ & Rango reducido \\
\hline NNFI & 0,956 & $\geq 0,9$ \\
\hline IFI & 0,969 & $\geq 0,9$ \\
\hline CFI & 0,968 & $\geq 0,9$ \\
\hline Chi-cuadrado normada & 1,1922 & $>1 ;<2$ \\
\hline AIC & $-46,85$ & Valores pequeños \\
\hline
\end{tabular}

Tabla 4. Índices de bondad de ajuste del modelo 1

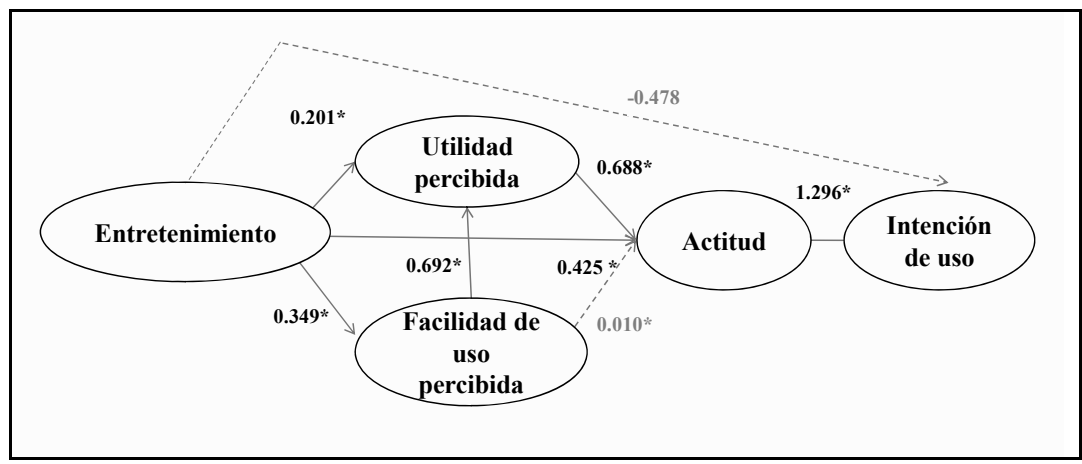

Gráfico 2. Modelo estimado «estudiante EEES» (N=165 casos) 


\begin{tabular}{|l|c|c|}
\hline \multicolumn{1}{|c|}{ Índice } & Valor & Valor recomendado \\
\hline Chi-cuadrado de Satorra-Bentler & $\mathrm{P}=0,048$ & $\mathrm{p} \geq 0,05$ \\
\hline RMSEA & 0,058 & $\leq 0,05$ \\
\hline Intervalo de confianza del RMSEA & $(0,032,0,080)$ & Rango reducido \\
\hline NNFI & 0,911 & $\geq 0,9$ \\
\hline IFI & 0,937 & $\geq 0,9$ \\
\hline CFI & 0,935 & $\geq 0,9$ \\
\hline Chi-cuadrado normada & 1,54 & $>1 ;<2$ \\
\hline AIC & $-26,31$ & Valores pequeños \\
\hline
\end{tabular}

Tabla 5. Índices de bondad de ajuste del modelo 2

\section{Discusión de resultados}

A nivel global, la evaluación de la experiencia ha sido muy positiva, tanto por parte de los alumnos encuestados, como por parte de los docentes que han intervenido. Además, puede decirse que la motivación del estudiante de las distintas asignaturas analizadas se ha visto reforzada positivamente, ya que las tecnologías le han permitido una mayor participación e involucración en los contenidos a emplear, en las tutorías, en el conocimiento previo del calendario y envío programado de actividades, así como en la comunicación y colaboración con otros estudiantes, de forma similar a como hacen en las redes sociales privadas. Además, las herramientas de comunicación han resultado muy útiles y valoradas positivamente por los alumnos, en especial el empleo de los foros para tutorías.

En lo que respecta a las variables analizadas en el contexto del modelo de aceptación de la tecnología, se han contrastado una serie de diferencias significativas entre los estudiantes de Diplomatura/Licenciatura (pre-EEES), y Grado (EEES). En primer lugar, se observó una evolución positiva respecto a la intención de uso de la asignatura (de 5,27 a 6,05), pero la variable entretenimiento (en la totalidad de sus ítems), fue peor valorada por los alumnos de Grado. Esto puede deberse a que, como se comentó anteriormente, los estudiantes pertenecen a una nueva generación (generación Net o Millennials), que vienen utilizando las tecnologías de forma más sofisticada y pasan gran parte de su tiempo libre utilizando videojuegos online y contenido multimedia. Es decir, llegan a la universidad con un perfil diferente, siendo consumidores de tecnología y utilizando distintas herramientas de forma que las generaciones anteriores no son capaces de entender (Junco y Mastrodicasa, 2007). Este hecho puede provocar que sus expectativas respecto al entretenimiento sean bastante elevadas, y 
finalmente no sean satisfechas, ya que Moodle es una plataforma de contenido fundamentalmente estático y orientada a la docencia.

Respecto a la estimación del modelo TAM con ambos tipos de estudiantes, se observó en primer lugar que las relaciones planteadas en dicho modelo se contrastaban en la muestra analizada. Por tanto, puede afirmarse que la intención de uso de la plataforma Moodle viene determinada fundamentalmente por la actitud del estudiante hacia la misma y esta a su vez se explica por la utilidad percibida por parte del estudiante y por la variable entretenimiento.

Por otra parte, se contrastaron diferencias interesantes en el efecto de la facilidad de uso en la actitud de los estudiantes. En los estudiantes de Diplomatura y Licenciatura, modelo estimado "estudiante pre-EEES", dicha variable mostraba un impacto directo en la actitud, lo que indica que era específicamente valorado por los alumnos el hecho de que la plataforma fuera sencilla de utilizar. No obstante, para los estudiantes de Grado, modelo estimado «estudiante EEES», más avanzados en el uso de tecnologías, dicha variable no era determinante de su actitud, sino que ejercía un impacto indirecto a través de la utilidad percibida, siendo estos resultados consistentes con los obtenidos por Teo, Lim y Lai (1999) al indicar que el uso de internet en un contexto de enseñanza-aprendizaje se considera fácil generalmente. Estos resultados son coherentes, también, con los obtenidos por Lee et al. (2005), que analizando una muestra de 554 estudiantes en una Universidad de Hong Kong, observaron que la facilidad de uso no mostraba un impacto directo en la actitud, sino que las variables que determinaban la misma eran la utilidad percibida y el entretenimiento.

Asimismo, se observó que el entretenimiento no ejercía un impacto directo en la intención de usar la plataforma en ninguno de los dos modelos estimados. Estos resultados resultan contrarios a los obtenidos por Lee et al. (2005) y Byoung-Chan et al. (2009), entre otros, que sí evidenciaron una relación directa entre el entretenimiento y la intención de uso en un entorno de enseñanza-aprendizaje. Dicho hallazgo puede deberse por una parte a que, como se ha comentado anteriormente, los estudiantes otorgaron puntuaciones muy bajas al entretenimiento proporcionado por la plataforma. Asimismo, al tratarse de una tecnología de uso obligatorio para seguir y superar las asignaturas, el entretenimiento solo va a jugar un papel indirecto en este caso. Por lo que su intención de usar la plataforma va a venir determinada por la actitud global hacia la misma, y en esta actitud sí que van a ejercer un papel clave la utilidad percibida (cómo la plataforma mejora el trabajo y facilita el aprendizaje), y también el entretenimiento que proporcione. 


\section{Conclusiones}

Los resultados de la experiencia realizada muestran que existe un nuevo perfil de alumnos, con un uso más avanzado e intensivo de las TI, y que demandan asimismo nuevas funcionalidades de la misma como herramienta de aprendizaje, que sea entretenida y motive su uso, que les muestre una utilidad patente, siendo a su vez sencilla de usar. Se ha comprobado que la aceptación y uso de la plataforma Moodle por parte de estudiantes de las asignaturas de Dirección de Empresas depende de su actitud hacia la plataforma, que viene determinada a su vez por su utilidad percibida y por el entretenimiento.

Puede afirmarse, por tanto, que la incorporación de las nuevas tecnologías a las aulas está cambiando los roles del docente, conforme a estudios previos (García-Varcárcel y Tejedor-Tejedor, 2011). Esto va a requerir el desarrollo de nuevos roles y habilidades por parte del docente. Vista la relevancia de variables como la utilidad percibida y el entretenimiento derivado del uso de la plataforma, el profesor precisa desarrollar los mencionados nuevos roles de gestor de contenidos y gestor de una comunidad.

Por ello, ante este nuevo escenario y estos nuevos retos, el profesorado precisa una formación técnico-didáctica, que incluya contenidos como diseño y producción de TI, recursos audiovisuales y multimedia e implantación de herramientas de social media (Cabero-Almenara, 2014), que le ayude a sacar el máximo partido a la tecnología como herramienta de apoyo en el proceso de enseñanza-aprendizaje.

Desde el punto de vista de los docentes, puede afirmarse que el trabajo realizado ha sido realmente enriquecedor, y se ha observado cómo los alumnos valoran muy positivamente la utilización del aula virtual, y aprecian las posibilidades que la misma ofrece para mejorar el proceso de aprendizaje, donde el papel desempeñado por el profesor es determinante. La utilidad de esta experiencia para los docentes se puede resumir en dos aspectos: 1) se ha comprobado cómo a pesar de los nuevos retos que plantea, la plataforma Moodle es una herramienta que les ayuda a organizar mejor el tiempo, que permite gestionar los contenidos de la materia y centralizar la información y comunicaciones con los alumnos; 2) se ha demostrado asimismo que el uso de las TI como elemento adicional en el proceso enseñanza-aprendizaje es además muy valorado por los estudiantes, lo que permite incrementar su participación e implicación en la asignatura, favoreciendo así el aprendizaje continuo y el desarrollo de las capacidades requeridas.

Finalmente, como cualquier trabajo de investigación, este no está exento de limitaciones. Se ha analizado una experiencia en una universidad 
concreta, por lo que la generalización de los resultados habría que tomarla con cautela. Sin embargo, sí que se han extraído conclusiones interesantes para aplicar la experiencia en otros contextos, reflexionando al respecto de los nuevos roles a desarrollar por el profesorado en el marco del EEES y las expectativas de este nuevo perfil de estudiantes en lo que se refiere al uso de las TI en la universidad, y en el ámbito docente, en particular. 


\section{REFERENCIAS BIBLIOGRÁFICAS}

Ahn, T., Ryu, S. y Han, I. (2007). The impact of web quality and playfulness on user acceptance of online retailing. Information \& Management, 44(3), 263-275.

Arbaugh, J. B., Godfrey, M. R., Johnson, M., Pollack, B. L., Niendorf, B. y Wresch, W. (2009). Research in online and blended learning in the business disciplines: Key findings and possible future directions. Internet and Higher Education, 12(2), 71-87.

Arias, D., Haro, C., Romerosa, M. M. y Navarro-Paule, A. J. (2010). Un enfoque innovador del proceso de enseñanza-aprendizaje en la dirección de empresas: el uso de simuladores en el ámbito universitario. Revista de Educación, 353, 707-721.

Byoung-Chan, L., Jeong-Ok, Y. e In, L. (2009). Learners' acceptance of elearning in South Korea: Theories and results. Computers \& Education, 53(4), 1320-1329.

Cabero-Almenara, J. (2014). Formación del profesorado universitario en TIC. Aplicación del método Delphi para la selección de los contenidos formativos. Educación XX1, 17(1), 111-132.

Cheong, J. H. y Park, M. Ch. (2005). Mobile internet acceptance in Korea. Internet Research, 15(2), 125-140.

Chung, J. y Tan, F. B. (2004). Antecedents of perceived playfulness: an exploratory study on user acceptance of general information-searching websites. Information \& Management, 41(7), 869-881.

Davis, F. D. (1989). Perceived usefulness, perceived ease of use and user acceptance of information technology. MIS Quarterly, 13(3), 319-340.
Fishbein, M. y Ajzen, I. (1975). Belief attitude, intention and behavior: an introduction to theory and research. Reading. MA: Addison-Wesley.

Florido, C.; Jiménez, J. L. y Santana, I. (2011). Obstáculos en el camino hacia Bolonia: efectos de la implantación del Espacio Europeo de la Educación Superior (EEES) sobre los resultados académicos. Revista de Educación, 354, 629-656.

García-Varcárcel, A. y Tejedor-Tejedor, F. J. (2011). Variables TIC vinculadas a la generación de nuevos escenarios de aprendizaje en la enseñanza universitaria. Aportes de las curvas roc para el análisis de diferencias. Educación XX1, 14(2), 43-78.

Greenberg, P. (2010). The impact of CRM 2.0 on customer insight. Journal of Business y Industrial Marketing, 25(6), 410-419.

Hair, J. F., Anderson, R. E., Tatham, R. L. y Black, W. C. (2004). Análisis multivariante. (5. ${ }^{\mathrm{a}}$ ed.). Madrid: Pearson Prentice Hall.

Hsiao, C. H. y Yang, C. (2011). The intellectual development of the technology acceptance model: A co-citation analysis. International Journal of Information Management, 31, 128-136.

Ives, B. y Jarvenpaa, S. L. (1996). Will the Internet revolutionize business education and research? Sloan Management Review, 37(3), 33-41.

Junco, R. y Mastrodicasa, J. (2007). Connecting to the net.generation: What Higher Education Professionals need to Know about Today's Students. Washington D. C.: NASPA.

Lara, P. (2005). Gestión de contenidos en la universidad: nuevos mercados, nue- 
vos retos, nuevos roles. Revista de Universidad y Sociedad del Conocimiento, 2(2), 54-56.

Lee, K. O. M., Cheung, C. M. K. y Chen, Z. (2005). Acceptance of internet-based learning medium: The role of extrinsic and intrinsic motivation. Information \& Management, 42(8), 1095-1104.

Liao, Ch. H. y Tsou, Ch. W. (2009). User acceptance of computer-mediated communication: The SkypeOut case. Expert Systems with Applications, 36(3), 4595-4603.

Lin, C. S., Ray, S. W. y Tsai, J. (2005). Integrating perceived playfulness into expectation-confirmation model for web portal context. Information \& $M a$ nagement, 42(5), 683-693.

Lonn, S. y Teasley, S. D. (2009). Saving time or innovating practice: Investigating perceptions and uses of Learning Management Systems. Computers \& Education, 53(3), 686-694.

Martín-Blas, T. y Serrano-Fernández, A. (2009). The role of new technologies in the learning process: Moodle as a teaching tool in Physics. Computers \& Education, 52(1), 35-44.

Martínez, E.; Fossas, M. y Cobo, F. B. (2010). De profesor a Community Manager: El Potencial Transformador de Moodle en las Relaciones Docente-Alumnos. Revista RELADA, 4(2), 112-121.

Martins, L. L. y Kellermanns, F. W. (2004). A model of business school students' acceptance of a web-based course management system. Academy of Management Learning and Education, 3(1), 7-26.

Moodle (2014). Moodle Statistics. Recuperado de: http://moodle.org/stats/

Moon, J. W. y Kim Y. G. (2001). Extending the TAM for a World-Wide-Web context. Information \& Management, 38(4), 217-230.

Oh, S. H., Kim, Y. M., Lee, Ch. W., Shim, G. Y., Park, M. S. y Jung, H. S. (2009). Consumer adoption of virtual stores in Korea: Focusing on the role of trust and playfulness. Psychology and Marketing, 26(7), 652-668.

Padilla-Meléndez, A.; Garrido-Moreno, A. y Del Águila-Obra, A. R. (2008). Factors affecting e-collaboration technology use among management students. Computers \& Education, 51(2), 609-623.

Romero, C.; Espejo, P. J.; Zafra, A.; Romero, J. R. y Ventura, S. (2013). Web Usage Mining for Predicting Final Marks of Students That Use Moodle Courses. Computer Applications in Engineering Education, 21(1), 135-146.

Satorra, A. y Bentler, P. M. (2001). A Scaled Difference Chi-square Test Statistic for Moment Structure Analysis. Psychometrika, 66(4), 507-514.

Schoonenboom, J. (2014). Using an adapted, task-level technology acceptance model to explain why instructors in higher education intend to use some learning management system tools more than others. Computers \& Education, 71, 247-256.

Suárez-Rodríguez, J. M.; Almerich, G.; Gargallo-López, B. y Aliaga, F. M. (2013). Las competencias del profesorado en TIC: estructura básica, Educación $X X 1,16(1), 39-62$.

Teo, T. S. H., Lim, V. K. G. y Lai, R. Y. C. (1999). Intrinsic and extrinsic motivation in internet usage. Omega. The International Journal of Management Science, 27(1), 25-37.

Terzis, V. y Economides, A. A. (2011). The acceptance and use of computer based assessment. Computers \& Education, 56, 1032-1044. 
Venkatesh, V. (2000). Determinants of Perceived Ease of Use: Integrating Control, Intrinsic Motivation, and Emotion into the Technology Acceptance Model. Information Systems Research, 11(4), 342-365.

Venkatesh, V. y Bala, H. (2008). Technology Acceptance Model 3 and a Research Agenda on Interventions. Decision Sciences, 39(2), 273-315.

Venkatesh, V. y Davis, F. D. (2000). A theoretical extension of the Techno- logy Acceptance Model: Four longitudinal field studies. Management Science, 46(2), 186-204.

Venkatesh, V.; Speier, C. y Morris, M. G. (2002). User acceptance enablers in individual decision making about technology: Toward an integrated model. Decision Sciences, 33(2), 297-316.

Zhang, P. y Li, N. (2005). The importance of affective quality. Communications of the ACM, 48(9), 105-108. 


\section{PERFIL ACADÉMICO Y PROFESIONAL DE LOS AUTORES/AS}

Antonio Padilla Meléndez, Doctor en Administración y Dirección de Empresas y Profesor Titular de Universidad de Organización de Empresas en la Universidad de Málaga. Ha sido responsable de diversos proyectos de investigación y de transferencia con varias empresas, así como ponente invitado en numerosas jornadas y congresos. Sus líneas de investigación son el emprendimiento académico, la innovación organizativa y el estudio de la aceptación y uso de la tecnología.

Ana Rosa del Águila Obra, Doctora en Administración y Dirección de Empresas y Profesor Titular de Universidad de Organización de Empresas en la Universidad de Málaga. Es Vicedecana de Calidad de la Facultad de Estudios Sociales y del Trabajo. Ha sido responsable de diversos proyectos de investigación. Sus líneas de investigación son la calidad de servicio, la innovación y el estudio de la aceptación y uso de la tecnología.

Aurora Garrido Moreno, Doctora en Administración y Dirección de Empresas y Profesor Ayudante Doctor de Organización de Empresas en la Universidad de Málaga. Sus líneas de investigación son la implementación de estrategias de gestión de relaciones con clientes (CRM) y Social Media, la innovación y el estudio de la aceptación y uso de la tecnología.

Dirección de los autores: Facultad de Estudios Sociales y del Trabajo Campus de Teatinos (Ampliación)

29071 Málaga

e-mail: apm@uma.es

anarosa@uma.es

agarridom@uma.es

Fecha Recepción del Artículo: 14. Marzo. 2012

Fecha Aceptación del Artículo: 05. Junio. 2012

Fecha Revisión para publicación: 15. Mayo. 2014 
